

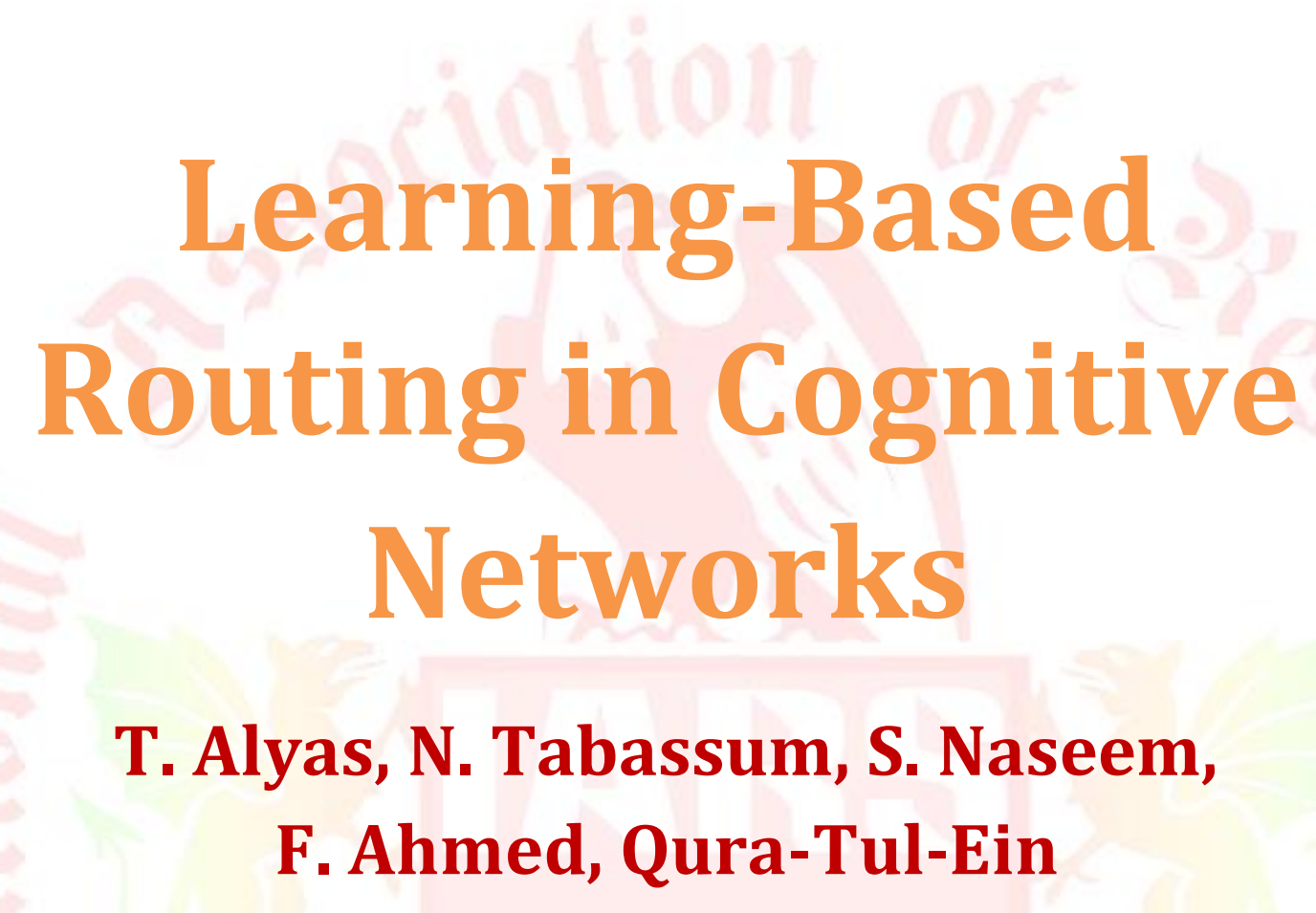




\title{
Learning-Based Routing in Cognitive Networks
}

\begin{abstract}
A BSTRACT - Intelligent Routing can influence the overall performance of a communication network's throughput and efficiency. Routing strategies is required to adapt to changing network loads and different topologies. Learning from the network environment, in order to optimally adapt the network settings, is an essential requirement for providing efficient communication services in such environments. Cognitive networks are capable of learning and reasoning. They can energetically adapt to varying network conditions in order to optimize end-to-end performance and utilize network resources. In this paper we will focus machine learning in routing scheme that includes routing awareness, a routing reconfiguration.
\end{abstract}

\section{KEY WORDS: ROUTING STARTEGIES, COGNITIVE NETWORK, PACKET ROUTING, NETWORK, DIRECTED ACYCLIC GRAPHS}

\section{INTRODUCTION}

Cognitive networking is an promising paradigm that deals with how heterogeneous systems learn relationships among network parameters, network events, and observed network performance, plan and make decisions in order to achieve local, end-to-end, and network-wide performance as well as resource management goals. Cognitive wireless networks are capable of reconfiguring their infrastructure, based upon experience, in order to adapt to continuously changing network environments. Cognitive networks are seen as a main facilitator of future heterogeneous internetworking and management, capable of continuously adapting to fluid network characteristics.

The term "cognitive network" has different interpretations with different emphasizes on the node behavior, operational objective, or the scope of the target problem Cognitive Radio (CR), with the ability to observe the surrounding network environment and reconfigure to adapt to network changes, is one of the most promising solutions. The core of cognitive radio as described by Mitola is the cognitive cycle, which consists of six processes observe, orient, plan, decide, act, and learn [1] Among diverse wireless technology supporting Internet access and other stream traffic services, a different vision is to integrate different wireless systems/networks and to appropriately use one of them based on the communication environments and the application requirements, based on reconfigurable communication and networking. Cognitive radio pioneered by J. Mitola 
III from software defined radio (SDR) was originally considered to improve spectrum utilization. [2].

\section{DIFFERENT MACHINE LEARNING METHODS FOR PACKET ROUTING}

\section{Q-ROUTING}

Q-Learning is a reinforcement learning algorithm that is able to learn an optimal sequence of actions in an environment which maximizes rewards received from the environment. Q-Routing is an adaptation from Q-Learning that is able to distributively route packets in a network.

\section{ANT-BASED ROUTING}

Ant-Based Routing is a novel variation of reinforcement learning that is based on simple biological "ants". These "ants" explore the network and rapidly learn optimal routes inspired by the stigmergy model of communication observed in ant colonies. This algorithm is more resilient than traditional routing algorithms, in the sense that random corruption of routes has limited effect on the computation of the packet routes.

\section{PARTICLE SWARM ROUTING}

Particle swarm optimization is a population based stochastic optimization technique developed by Dr. Russ Eberhart and Dr. James Kennedy in 1995, inspired by social behavior of bird flocking or fish schooling. Particle Swarm Routing is initialized with a group of random solutions and then searches for optima by updating generations [3].

\section{PROPOSE NETWORK MODEL}

We will design such cognitive function which can learn the demand base request and discover more-optimal paths and update our repository. Designing the machine learning base routing algorithm in heterogeneous networks is a big challenge. In a cognitive network, the performance of routing links belonging to different networks is quite different. The complex heterogeneous network environment often varies. Consistent Link management, transmission data rate and reliability change with the environment. In an overlapped network scenario it is very difficult to predict and control spectrum interference of wireless links. Routing strategy in cognitive network is affected by factors such as the ability to access multiple networks, throughput, user preference, Quality of service requirements, and location. 


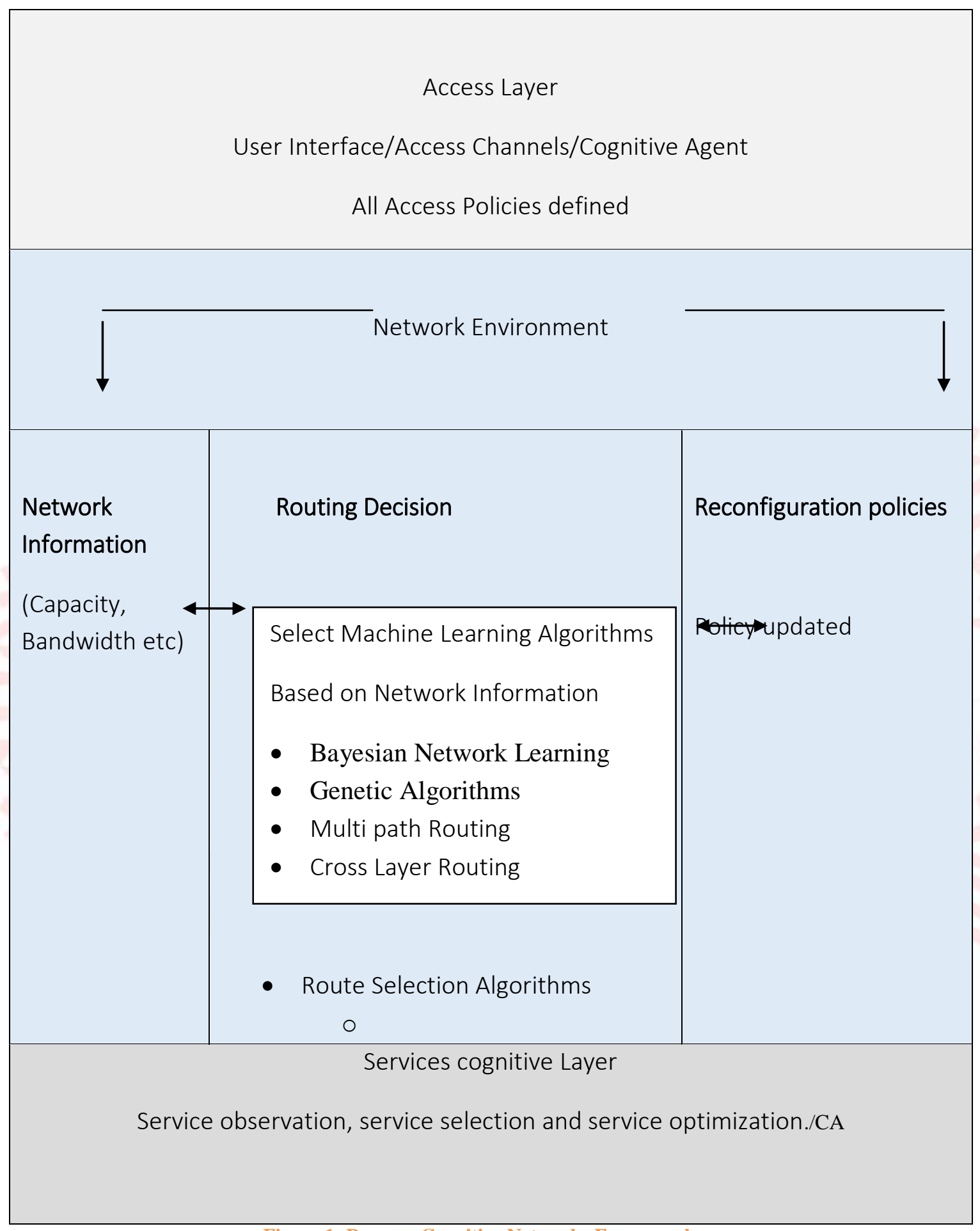

Figure 1: Propose Cognitive Networks Framework 
The Different cognitive design based functions can be implemented as distributed intelligent agents. Agents with different machine learning algorithms and having reasoning capabilities can be deployed on each layer in the network to monitor and collect network environment information. These functions cooperate and exchange information so that the complex network can perceive its current status. In Fig. 1 if we known the entire network environment, in certain or uncertain machine learning algorithms, and select the best possible route and reconfigurable policy has been determined and appropriate services are triggered.

End-to-end targets can be achieved by knowing the current network status, and network resources can be evaluated, predicted environment, planned, adjusted, and allocated based on a knowledge library by cognitive agents. As a result, the network can be selflearning, self-optimization, self-perception, and self-configuration capabilities.

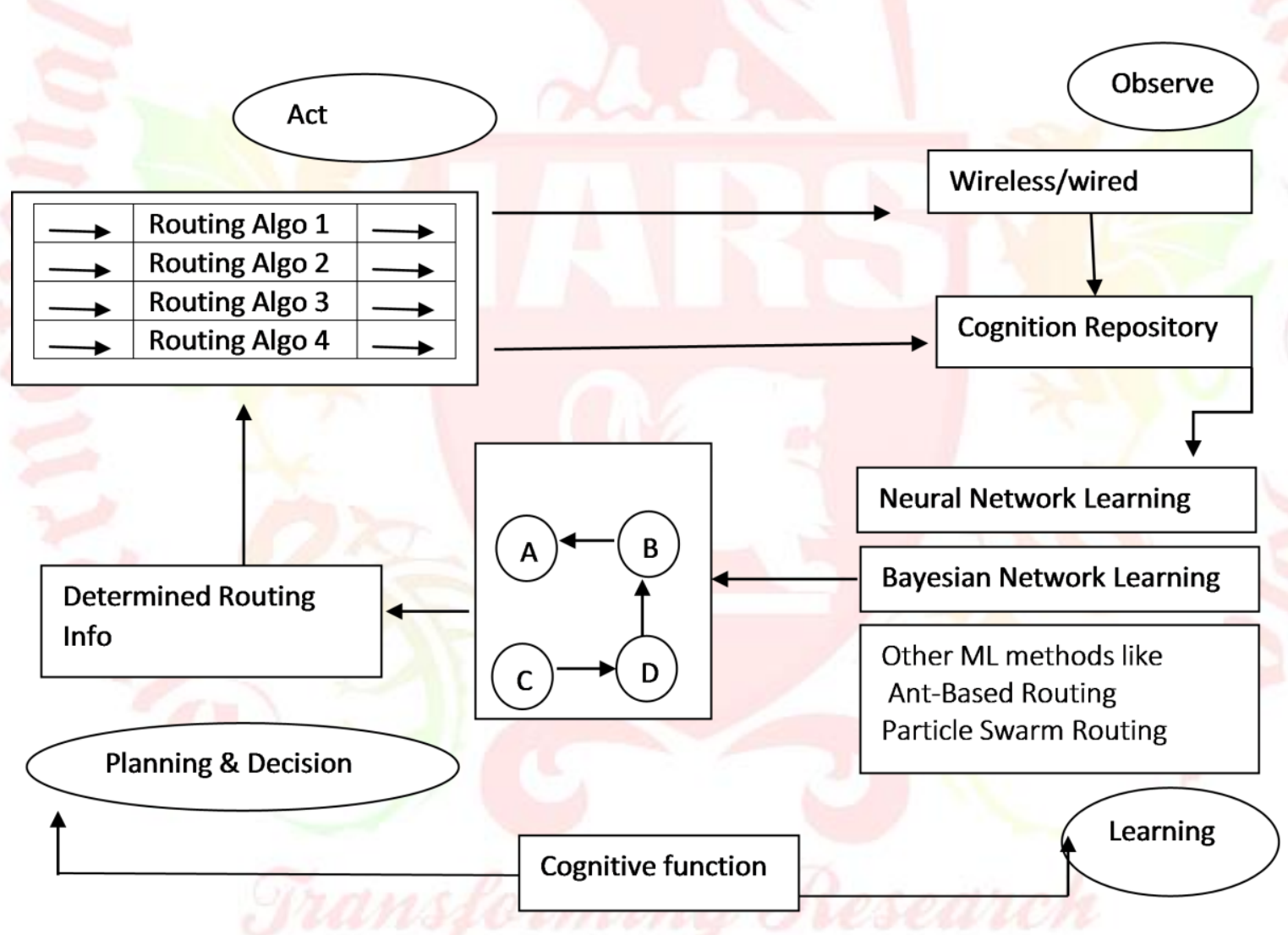

Figure 2: Proposed Learning in Cognitive Network

In Complex network, there is no mechanism to infer when congestion is to about occur and therefore it waits till some important packets are lost for reacting to face congestion 
in the network. In fig. 2 suppose that our network is facing congestion, and then Cognitive Function will be work like brain of the cognitive network node where the optimization decisions for protocols within the layer are made.

\section{LEARNING THROUGH BAYESIAN NETWORK}

A Bayesian Network is a probabilistic model in the form of directed acyclic graphs (DAG) that represents a set of random variables by its nodes and their correlations by its edges. Bayesian Networks has an advantage that they visually represent all the relationships between the variables in the system via connecting arcs and they can handle situations where the data set is incomplete. In complex network there is uncertain and inconsistent data flow comes [4].

In our proposed model the cognitive Function realizes the Learn phase and Decide phases of the cognition cycle. The Learning phase is a key phase in the cognition process, where the cognitive node exploit the information collected in the observe phase to infer a probabilistic arrangement that connects the parameters of interest, using the Bayesian model and predict the network.

This prediction can be very important in the Plan and Decide phase to optimize a controllable parameter, or to predict an unwanted behavior of the network, e.g., congestion, and take the necessary actions in the protocol stack before this happens. Finally, in the Act phase, the decisions made in the Plan and Decide phase are effected, e.g., controllable parameters are modified to optimize the network performance.

\section{CONCLUSION}

In evolving different networks, network configuration cannot be optimized manually. The coexistence of heterogeneous networks also brings a more complicated networks environment. Cognitive technology provides a way of configuring networks dynamically, and best optimizing in performance, and user resources. In this paper, a cognitive routing scheme for heterogeneous networks has been proposed. This scheme involves a routing algorithm frame composed of a situation awareness, route reconfiguration by learning element in cognitive function. 


\section{REFERENCES}

[1]. Friend, Daniel H. "COGNITIVE NETWORKS: FOUNDATIONS TO APPLICATIONS." Dissertation, Blacksburg, Virginia, 2009.

[2]. K. -C. Chen, Y. -J. Peng, N. Prasad. "COGNITIVE RADIO NETWORK ARCHITECTURE". National Science Council, Taiwan, 2008.

[3]. Edwill Nel, C.W. Omlin. "MACHINE LEARNING ALGORITHMS FOR PACKET ROUTING IN TELECOMMUNICATION NETWORKS." Bellville, South Africa.

[4]. Giorgio Quer, Hemanth Meenakshisundaram, Bheemarjuna. "USING BAYESIAN NETWORKS FOR COGNITIVE CONTROL OF MULTI-HOP WIRELESS NETWORKS." The 2010 Military Communications Conference, 2010: 6. 
